\title{
Reasoning about Skills and Competencies
}

\author{
Maryam Fazel-Zarandi ${ }^{1}$ and Mark S. Fox ${ }^{2}$ \\ ${ }^{1}$ Department of Computer Science, \\ ${ }^{2}$ Department of Mechanical and Industrial Engineering, \\ University of Toronto, Toronto, Canada \\ mfazelecs.toronto.edu, msfeeil.utoronto.ca
}

\begin{abstract}
Human Resources Management (HRM) is the strategic management of the employees, who individually and collectively contribute to the achievement of the strategic goals of an organization. Most HRM tasks are based on acquiring the right information and reasoning about skills and competencies of individuals. In this paper we present a formal ontology for competency management and consider three reasoning problems related to HRM, namely, determining the set of skills of an individual, conducting competency gap analysis, and determining whether an individual satisfies a set of requirements.
\end{abstract}

Keywords: Competency management, Skills ontology.

\section{Introduction}

In today's competitive business environment, companies need to accurately grasp the competency of their human resources in order to be successful. This is particularly important for organizations that engage with multiple and changing clients such as consulting firms and software development companies since these organizations need to be able to flexibly respond to internal and external demands for skills and competencies. As such, proper and flexible human resources management (HRM) and competency management approaches for more effective resource allocation, human resource hiring, knowledge management, learning support, and human resource development are of outmost importance to the survival of these companies.

In order to facilitate the management of available human resources' competencies, provide a global view of competencies available at the organizational level, and perform qualitative and quantitative reasoning about available and required skills, the use of formal ontologies has been suggested in previous research. In almost all the existing systems and approaches, however, the focus has been either on building and maintaining ontology-based skill catalogs [e.g. 1, 2, 5, 6], or searching for individuals that match certain requirements [e.g. 2, 3, 10, 11, 12]. As such, the reasoning has been limited to subsumption checking. Furthermore, existing approaches have mainly focused on binary matching and do not take into account the cases where skills do not completely match existing requirements.

In this paper, we attempt to extend existing ontologies to address other interesting HR-related problems in addition to determining whether an individual satisfies a set of requirements. These problems include: 1) determining the set of skills of an 
individual and 2) conducting competency gap analysis to determine who to train and what training program to offer. Note that in this paper we are not addressing the concerns raised about incorporating the human and judgmental aspects of HR.

\section{Motivating Use-Cases}

Following the ontology development methodology of Gruninger and Fox [8], we first identify the problems the ontology is going to address. The following examples from two different perspectives motivate and illustrate the need for the skills ontology.

- Example 1: A HR manager working at a high tech firm is responsible for suggesting individuals for a team to work on a specific software development project. To do her job well, she needs to know the competencies of available human resources to match them with existing requirements, to determine the training and development needs of employees, and to determine if the company suffers from a shortage of competency. As such, she has asked all the employees to state their skills based on an existing skills taxonomy which consists of technical computer skills related to all aspects of software design and development. However, since self declarations of skills and competencies can be inaccurate or insufficient, she wants to be able to distinguish between proposed and demonstrated skills of an individual and detect the competencies that were not explicitly mentioned but the individual may have. In addition, she wants to determine the gaps that may exist between the skills of an individual and a set of requirements and use this information to suggest under-qualified matches when qualified ones cannot be found or to suggest training opportunities.

- Example 2: A software engineer with previous work experience working at two high tech firms is looking for a software development position that includes some managerial elements. He has a degree in computer science and has taken some management courses. To make a decision, he needs to find out which job postings he is currently qualified for, what are the requirements of the position he wants, and what training is required for him to qualify for that particular job.

Using these examples we can define the ontologies' requirements in the form of questions that it must be able to answer. We divide these questions into those that previous research has been able to address, and those that it does not address.

- Questions addressed by previous research: What are the credentials and previous work experience of an individual? Who are the individuals that satisfy a particular requirement? What are the proposed skills of an individual? Which skills are required for a particular job? Who are the qualified matches for a job posting? Which job postings is an individual qualified for?

- Questions not addressed by previous research: What are the demonstrated skills of an individual? What are the skills that an individual has which were not explicitly mentioned? Who are the individuals that may have a particular skill? Does the company suffer a shortage of a competency? If qualified matches cannot be found, who are the next best choices? If an individual does not qualify for a particular job, what training is required? 


\section{The Skills Ontology}

In this section we briefly present the ontology we developed to answer the questions posed in section $2^{1}$. We use first-order logic (FOL) as the basis for the ontology because of its expressive and declarative capability. With the ontology being defined in FOL and applied in a theorem prover, the ontology will have the deductive capability to answer queries. In particular, our ontology is an extension of the Process Specification Language (PSL) [9], a first-order language for modeling processes.

\subsection{Skill}

There are several definitions of competency present in the literature [4]. The definition we assume is the one given by the HR-XML Consortium work group (http://hrxml.org): "a specific, identifiable, definable, and measurable knowledge, skill, ability and/or other deployment-related characteristic (e.g. attitude, behavior, physical ability) which a human resource may posses and which is necessary for, or material to, the performance of an activity within a specific business context." We adopt this definition for its emphasis on measurable knowledge and skills and the connection between competencies and activity performance. Hereafter, we focus on measurable skills possessed by human resources and use skill instead of competency.

We assume skills in a specific domain of interest and distinguish between demonstrated and proposed skills. Demonstrated skills are those related to previous performed activities. Proposed skills are either based on self-declarations of expertise or the skills that an individual may posses after completing a learning activity.

As stated earlier, a skill suggests the possibility of performing an activity. In the formal PSL ontology, the notion of activity is a basic construct, which corresponds intuitively to a kind of activity. An activity may have associated occurrences, which correspond to individual instances or executions (from start to finish) of the activity ${ }^{2}$. We represent the relation between a skill and an activity using the enables predicate:

$$
\ldots s \operatorname{Skill}(s) \equiv \mid a \operatorname{Activity}(a) . \operatorname{enables}(s, a)
$$

We define specific tools or technologies as resources in PSL and associate them with activities using the requires relation. For example, in "object-oriented coding in Java", "object-oriented coding" is the activity which requires "Java". We also define the related-to relation between two skills indicating that they are highly related to each other in the domain of interest (i.e., having one usually implies having the other). For example, software development skills are related-to programming skills. Put formally, two skills are related if they enable the same activity, or if they enable subactivities of the same activity:

$$
\begin{aligned}
& \ldots s 1, s 2 \text { related-to }\left(s_{1}, s_{2}\right) \equiv \mid a \operatorname{Activity}(a) .\left[\left(\operatorname{enables}\left(s_{1}, a\right) .\right.\right. \\
& \left.\quad \text { enables }\left(s_{2}, a\right)\right)-\left(\mid a_{1}, a_{2} \operatorname{Activity}\left(a_{1}\right) . \operatorname{Activity}\left(a_{2}\right) . \operatorname{subactivity}\left(a_{1}, a\right) .\right. \\
& \left.\left.\quad \text { subactivity }\left(a_{2}, a\right) . \operatorname{enables}\left(s_{1}, a_{1}\right) . \operatorname{enables}\left(s_{2}, a_{2}\right)\right)\right]
\end{aligned}
$$

${ }^{1}$ For brevity, not all axioms of the ontology are included in this paper. The interested reader is referred to http://www.cs.toronto.edu/ mfazel/publications/mfz_fox_pro-ve_extended.pdf .

${ }^{2}$ In PSL an activity is not a class or type with occurrences as members; rather, an activity is an object, and occurrences are related to this object by the binary predicate occurrence-of. 


\subsection{Learning Activity and Work Experience}

A learning activity (LA) is an activity that has one or more learning outcomes associated with it and occurs within a particular context [7]. A learning outcome is what the learners should be able to do after completing the LA. Currently we only consider formal learning activities which occur as a result of instructor-led programs within the curricula of educational institutions or the courses or workshops offered by different agencies [15]. Formal learning activities can have a set of skills at a particular knowledge level (beginner, intermediate, advanced, expert) as required preconditions (hasprecondition), but must have at least one proposed skill at a particular knowledge level as outcome (has-outcome).

$$
\ldots x \text { FormalLearningActivity }(x) \equiv \operatorname{Activity}(x) . \mid y, z \text { has-outcome }(x, y, z)
$$

An individual has taken a learning activity if and only if an occurrence of that activity exists in which she has participated in:

$$
\begin{aligned}
& \ldots x, y, t, e \text { has-taken }(x, y, t, e) \equiv(\operatorname{Person}(x) . \text { FormalLearning } \operatorname{Activity}(y) . \\
& \mid o \text { occurrence-of }(o, y) \cdot \operatorname{participates-in}(x, o) . \operatorname{beginof}(o)=t . \quad \operatorname{endof}(o) \\
& \quad=e \cdot \operatorname{legal}(o))
\end{aligned}
$$

Having the definition for formal learning activity, we can define a degree in a field of study as a program that requires a set of formal learning activities (requires-fla), is offered by some organization (offered-by), and has a starting and ending time point. If an individual has a degree then it must be the case that she has completed all the formal learning activities required for the degree.

Work experience indicates past performed activities at a particular organization. We associate these activities to positions the experience refers to using the includesactivity relation. We define the has-experience predicate between a person, a position, an organization, and a starting and ending time point. If an individual has a previous work experience then she has participated in an occurrence of all the activities associated with the position that experience refers to:

$$
\begin{aligned}
& \ldots x, y, p, t, e \text { has-experience }(x, p, y, t, e) \theta(\operatorname{Person}(x) . \operatorname{Position}(p) . \\
& \text { Organization }(y) .(\ldots a \text { includes-activity }(p, a) \theta(\mid o \text { occurrence-of }(o, a) . \\
& \quad \text { participates-in }(x, o) . \text { legal }(o))))
\end{aligned}
$$

We also define has-experience-in between a person, a skill, and a positive integer to denote the years of experience the individual has with respect to a skill. For brevity we omit the axioms related this predicate.

\subsection{Job Posting}

We define a job posting as a set of requirements in terms of job related descriptions and constraints on skills. Every job posting has a description (has-description), a set of skill requirements (has-requirement), and zero or more degree requirements (hasdegree-requirement). Skill requirements can either be must-have or nice-to-have constraints. 
$\ldots j \operatorname{JobPosting}(j) \equiv \mid d, r, z$ has-description $(j, d)$. Requirement $(r)$.

has-requirement $(j, r, z) .(z=$ must-have $-z=$ nice-to-have $)$

Requirements are constraints on proposed or demonstrated skills. Every requirement refers to only one skill. It is possible to defined requirements by adding constraints on minimum required years of experience performing activities related to a skill (requires-experience), required knowledge level (requires-knowledge), or required tool or technology (requires-use). For example, a resource requirement requires the use of one or more tools or technologies.

The description consists of a title (has-title), a position (has-position) including the specific activities that the individual holding the job needs to perform, a brief description in free text format (has-brief-description), and a category (has-category).

\section{Additional Axioms}

\subsection{Determining the Set of Skills}

Using the HR-XML definition, having a particular skill becomes tightly bound to the evidence that suggests one has a certain skill at a particular level of proficiency. The evidence also helps in understanding how a skill can be achieved, which is especially useful for arranging training programs. To detect whether an individual has a skill, we also need to determine the particular level of proficiency which may depend on different factors such as the span of the activities one can perform, how much experience one has in performing an activity, how well an activity is performed, what others think about how an activity was performed, etc. Different quantitative and qualitative measurement scales exist for evaluating an individual against a skill. Rating scales are the most popular and consist of a numeric scale with a brief description of each number's corresponding meaning. The disadvantage of these scales, however, lies in their inconsistent interpretations across users of a scale [13]. To overcome this problem, we distinguish between skills enabling two different types of activities: 1) activities which successfully performing them confirms proficiency in a skill (e.g., brain surgery); and 2) activities which the outcome does not necessarily confirm proficiency (e.g., having programmed once does not mean one is a programmer). We focus only on the first type for demonstrated skills and plan on addressing the second type in future by considering social networks and peer reviews.

To determine the skill set of an individual, we use learning activities and experiences related to the workplace and consider demonstrated and proposed skills. We define three relations: has-skill, has-proposed-skill, and may-have-skill.

If an individual can perform all the activities which a skill enables, then it is the case that she has that skill:

$$
\begin{aligned}
& \ldots x, y \text { has-skill }(x, y) \equiv(\operatorname{Person}(x) . \operatorname{DemonstratedSkill}(y) .(\ldots a \text { enables }(y, a) \\
& \theta \mid o \text { occurrence-of }(o, a) . \operatorname{participates-in}(x, o) . \operatorname{legal}(o)))
\end{aligned}
$$

Considering learning activities, if a person has taken a formal learning activity and a proposed skill is a precondition or outcome of it, then it is proposed that the person has that skill: 
$\ldots x, y, t, e, z, k$ has-taken $(x, y, t, e)$. [has-outcome $(y, z, k)-$ has-precondition $(y, z, k)] \theta$ has-proposed-skill $(x, z, k)$

If an individual has participated in an occurrence of an activity which required a particular resource (tool or technology), then it might be the case that she can perform other activities which require the same resource. For example, if an individual has experience plotting graphs in Microsoft Excel, then she might also be able to do macro programming in Excel.

$$
\begin{gathered}
\ldots a, a^{\prime}, r \text { requires }(a, r) . \operatorname{requires}\left(a^{\prime}, r\right) \theta(((\mid o \text { occurrence-of }(o, a) . \\
\text { participates-in }(x, o) . \operatorname{legal}(o)) .\left(\neg \mid o^{\prime} \operatorname{occurrence-of}\left(o^{\prime}, a^{\prime}\right) .\right. \\
\text { participates-in } \left.\left.\left.\left(x, o^{\prime}\right) . \operatorname{legal}\left(o^{\prime}\right)\right)\right) \theta \text { may-perform }\left(x, a^{\prime}\right)\right)
\end{gathered}
$$

and as such have the skills that enable those activities:

$$
\begin{aligned}
& \ldots x, y \text { Person }(x) . \operatorname{Skill}(y) .(\mid a \text { enables }(y, a) . \text { may-perform }(x, a)) \theta \\
& ((\ldots z \text { enables }(y, z) \theta((\mid o \text { occurrence-of }(o, z) \cdot \operatorname{participates-in}(x, o) . \\
& \text { legal }(o))- \text { may-perform }(x, z))) \theta \text { may-have-skill }(x, y))
\end{aligned}
$$

We can also consider highly related skills in the domain of interest. For example, software development skills are related-to programming skills, and having one may suggest having the other.

$\ldots x, y, z$ has-skill $(x, y) . \neg$ has-skill $(x, z) \theta($ related-to $(y, z) \theta$ may-have-skill $(x, z))$

\subsection{Gap Analysis}

Competency gap analysis serves two purposes in HRM. First, in real world situations, it is rarely the case that individuals satisfy all the requirements of a job posting. Second, competency gap analysis is beneficial for understanding whether the company suffers from a shortage of knowledge or skill in a particular area, and for finding suitable learning opportunities and arranging training programs [14]. To this end, we need to calculate the gap between the set of requirements and the set of skills of an individual. We consider two types of gap: competency gap and proficiency gap. A competency gap refers to the absence of a skill in the individual's profile.

$\ldots p, s, k$ has-competency-gap $(p, s) \equiv$

$$
\text { ( } \neg \text { has-skill }(p, s) . \neg \text { has-proposed-skill }(p, s, k) . \neg \text { may-have-skill }(p, s))
$$

A proficiency gap, on the other hand, refers to the difference in proficiency levels of a skill requirement and a skill statement. To determine proficiency gap for demonstrated skills, we focus on metrics such as years of experience and activities that an individual can perform. It is possible to define domain-specific metrics, such as the number of bugs found in a code for a programmer, or the number of failed projects for a contractor, etc. For example, a proficiency gap based on years of experience can be determined as follows:

$$
\begin{aligned}
& \ldots p, r, s, m, e \operatorname{Person}(p) . \operatorname{Requirement}(r) . \operatorname{refers-to}(r, s) . \\
& \text { requires-experience }(r, m) . \text { has-experience-in }(p, s, e) . e<m \theta \\
& \quad \text { has-proficiency-gap }(p, r)
\end{aligned}
$$




\subsection{Skills-Requirements Matchmaking}

In skills-requirements matchmaking, we are interested in determining whether or not an individual satisfies a set of requirements. An individual satisfies a requirement if and only if 1) when the requirement requires experience greater than zero, then she has demonstrated that skill for the require time period; otherwise, 2) she has the proposed skill.

$\ldots p, r, s, m, e$ satisfies $(p, r) \equiv \operatorname{refers-to}(r, s)$. requires-experience $(r, m)$.

$((m=0 \theta$ has-proposed-skill $(p, s))-(m \gamma 0 \theta$ has-experience-in $(p, s, e)))$

For a job posting $j$, a qualified match denotes that an individual satisfies all its degree and skill requirements.

$\ldots j, p((\ldots t, f$ has-degree-requirement $(j, t, f) \theta \exists d$ has-degree $(p, d)$.

has-degree-title $(d, t)$. has-study-field $(d, f))$.

$(\ldots r$ has-requirement $(j, r$, must-have) . satisfies $(p, r))) \theta$ qualified-match $(j, p)$

In real world situations, however, it rarely happens that applications match all the requirements specified in a job posting. For this, in addition to the qualified match, we consider different types of under-qualified matches. For one type of under-qualified match, we relax the required proficiency level constraints. In this case, an application is considered to be proficiency-under-qualified match for a job posting if and only if a proficiency gap exists for one or more of the required skills. Another type of underqualified match, competency-under-qualified match, takes competency gaps into account and considers all individuals who satisfy a subset of the required skills.

\section{Conclusions and Future Directions}

This paper presented a formal ontology for competency management and considered three problems related to HRM, namely, determining the set of skills of an individual, conducting competency gap analysis, and determining whether an individual satisfies a set of requirements. The main evaluation criterion for the development of the ontology was competency, the capability of a representation to support the use-cases for which it is designed. An interesting topic of future research would be to evaluate the ontology according to knowledge representation criteria such as consistency and completeness, as well as systems performance criteria like efficiency and scalability.

In addition to satisfying advertised job requirements, other factors such as job seekers' and recruiters' preferences, cultural fit, and ability to adapt to the company's marketplace play an important part in selecting employees. Furthermore, when considering individuals for teams, complexities may arise due to fitness between an individual and other team members. It would be interesting to see how these complexities can be supported by automated techniques.

Acknowledgments. The authors gratefully acknowledge the constructive comments and helpful advice of Professor Michael Gruninger. This research is supported, in part, by the Natural Science and Engineering Research Council of Canada. 


\section{References}

1. Biesalski, E., Abecker, A.: Human Resource Management with Ontologies. In: Althoff, K.-D., Dengel, A.R., Bergmann, R., Nick, M., Roth-Berghofer, T.R. (eds.) WM 2005. LNCS (LNAI), vol. 3782, pp. 499-507. Springer, Heidelberg (2005)

2. Bizer, C., Heese, R., Mochol, M., Oldakowski, R., Tolksdorf, R., Eckstein, R.: The Impact of Semantic Web Technologies on Job Recruitment Processes. In: Proceedings of the 7th International Conference Wirtschaftsinformatik (2005)

3. Colucci, S., Di Noia, T., Di Sciascio, E., Donini, F.M., Mongiello, M., Mottola, M.: A Formal Approach to Ontology-Based Semantic Match for Skills Descriptions. Journal of Universal Computer Science 9(12), 1437-1454 (2003)

4. De Coi, J., Herder, E., Koesling, A., Lofi, C., Olmedilla, D., Papatreou, O., Siberski, W.: A Model for Competence Gap Analysis. In: Proceedings of 3rd International Conference on Web Information Systems and Technologies (WEBIST) (2007)

5. Dittmann, L.: Towards Ontology-based Skill Management, Projektbericht zum Verbundprojekt KOWIEN, Universität Duisburg-Essen (2003)

6. Gomez-Perez, A., Ramirez, J., Villazon-Terrazas, B.: Reusing Human Resources Management Standards for Employment Services. In: Proceedings of the Workshop on the First Industrial Results of Semantic Technologies at ISWC/ASWC, pp. 28-41 (2007)

7. Gráinne, C., Fill, K.: A Learning Design Toolkit to Create Pedagogically Effective Learning Activities. Journal of Interactive Media in Education 8 (2005)

8. Gruninger, M., Fox, M.S.: The Design and Evaluation of Ontologies for Enterprise Engineering. In: Workshop on Implemented Ontologies, European Conference on Artificial Intelligence (ECAI 1994), Amsterdam, NL (1994)

9. Gruninger, M., Menzel, C.: Process specification language: Theory and applications. AI Magazine 24, 63-74 (2003)

10. Lau, T., Sure, Y.: Introducing Ontology-Based Skills Management at a Large Insurance Company. In: Proceedings of the Modellierung, pp. 123-134 (2002)

11. Liu, P., Dew, P.: Using Semantic Web Technologies to Improve expertise Matching within Academia. In: Proceedings of I-KNOW 2004, pp. 370-378 (2004)

12. Mochol, M., Wache, H., Nixon, L.: Improving the Accuracy of Job Search with Semantic Techniques. In: Abramowicz, W. (ed.) BIS 2007. LNCS, vol. 4439, pp. 301-313. Springer, Heidelberg (2007)

13. Moyer, R.: Defining and Measuring Competencies: An Overview of Approaches. Click2learn Inc. (2001)

14. Schmidt, A., Kunzmann, C.: Towards a Human Resource Development Ontology for Combining Competence Management and Technology-Enhanced Workplace Learning. In: Meersman, R., Tari, Z., Herrero, P. (eds.) OTM 2006 Workshops. LNCS, vol. 4278, pp. 1078-1087. Springer, Heidelberg (2006)

15. Schugurensky, D.: The Forms of Informal Learning: Towards a Conceptualization of the Field, NALL Working Paper No. 19 (2000) 